\title{
Displacement of Pile-Reinforced Slopes with a Weak Layer Subjected to Seismic Loads
}

\author{
Haizuo Zhou, ${ }^{1,2}$ Gang Zheng, ${ }^{1,2,3}$ Xinyu Yang, ${ }^{1,2}$ Yu Diao, \\ Lisen Gong, ${ }^{1,2}$ and Xuesong Cheng ${ }^{1,2}$ \\ ${ }^{1}$ School of Civil Engineering, Tianjin University, Tianjin 300072, China \\ ${ }^{2}$ Key Laboratory of Coast Civil Structure Safety, Tianjin University, Ministry of Education, Tianjin 300072, China \\ ${ }^{3}$ State Key Laboratory of Hydraulic Engineering Simulation and Safety, Tianjin University, Tianjin 300072, China \\ Correspondence should be addressed to Yu Diao; yudiao@tju.edu.cn
}

Received 30 May 2016; Accepted 14 August 2016

Academic Editor: Giovanni Garcea

Copyright (C) 2016 Haizuo Zhou et al. This is an open access article distributed under the Creative Commons Attribution License, which permits unrestricted use, distribution, and reproduction in any medium, provided the original work is properly cited.

\begin{abstract}
The presence of a weak layer in a slope requires special attention because it has a negative impact on slope stability. However, limited insight into the seismic stability of slopes with a weak layer exists. In this study, the seismic stability of a pile-reinforced slope with a weak thin layer is investigated. Based on the limit analysis theory, a translational failure mechanism for an earth slope is developed. The rotational rigid blocks in the previous rotational-translational failure mechanism are replaced by continuous deformation regions, which consist of a sequence of $n$ rigid triangles. The predicted static factor of safety and collapse mechanism in two typical examples of slopes with a weak layer compare well with the results obtained from the available literature and by using the Discontinuity Layout Optimization (DLO) technique. The lateral forces provided by the stabilizing piles are evaluated using the theory of plastic deformation. An analytical solution for estimating the critical yield acceleration coefficient for the pile-reinforced slopes is derived. Based on the proposed translational failure mechanism and the corresponding critical yield acceleration coefficient, Newmark's analytical procedure is employed to evaluate the cumulative displacement. Considering different real earthquake acceleration records as input motion, the effect of stabilizing piles and varying the spacing of piles on the cumulative displacement of slopes with a weak layer is investigated.
\end{abstract}

\section{Introduction}

Many catastrophic slope failures have been reported in the past due to earthquakes. Estimation of the stability of slopes subjected to seismic loads is a very important task in geotechnical engineering. There are two practical estimation methods that exist for this problem: the first one is calculating the factor of safety of slopes by considering pseudostatic earthquake body forces within a soil mass (e.g., Seed et al. [1]; Seed [2]; Chen [3]). The concept of factor of safety has been widely used because it is simple and straightforward extension of static considerations. However, it provides no details regarding the shaking process. On the other hand, the technique of slopes stabilized by reinforcement is widely employed by geotechnical engineers to enhance the stability of slopes. The pseudostatic approach may underestimate the stability of reinforced slopes for large earthquake acceleration in engineering design (Ling et al. [4]; Michalowski [5]).

The second one calculates the cumulative displacement subjected to seismic loads. The most common approach is Newmark's [6] sliding block method, which calculates the cumulative displacement of slopes by integrating earthquake acceleration in a one-block translational mechanism. This approach has the advantage of providing information during an earthquake and being less time consuming; it has been further extended to the rotational mechanism (e.g., Chang et al. [7]; Li et al. [8]) and multiblock mechanism (e.g., Michalowski [9]) of slope through limit analysis. Moreover, this approach has been employed for the seismic displacements of reinforced slopes by other researchers (e.g., Ling et al. [4]; Ling and Leshchinsky [10]; Michalowski and You [11]; He et al. [12]). 
In engineering practice, the existence of a weak layer in slopes requires special attention because the low shear strength of a weak layer has an adverse effect on the performance of a slope. The previous studies on slopes with a weak layer were performed under the static condition. Fredlund and Krahn [13] compared several methods for analyzing the stability of a nonhomogeneous soil slope with a weak layer. Moreover, finite element (FE) analysis, incorporated with the shear strength reduction technique, was applied to investigate the stability of a slope with a weak layer (Griffiths and Marquez [14]; Ho [15]). Based on the upper-bound method, Huang et al. [16] proposed a rotational-translational collapse mechanism to assess the factor of safety of slopes with a weak layer. Their analytical results were verified using the finite element method. However, few studies have been conducted regarding evaluating the seismic performance of slopes with a weak layer.

In this study, a translational failure mechanism is developed to evaluate the stability of slopes with a weak layer, which is validated using other solutions with respect to the static factor of safety. Furthermore, a pseudostatic method is employed within the limit analysis framework to calculate the critical yield acceleration coefficient of a reinforced slope. Finally, considering different real earthquake acceleration records as input motion, Newmark's analytical approach is used to assess the cumulative displacement of two typical cases of slopes with a weak thin layer.

\section{Critical Yield Acceleration Coefficient for Pile-Reinforced Slopes with a Weak Layer}

In limit analysis theory, soil is assumed to deform plastically according to the normality rule associated with the MohrCoulomb yield criterion (e.g., Lu et al. [17]). For homogeneous slopes, the rotational log-spiral failure mechanism has been found to be the most adverse for the stability of slopes (e.g., Chen [3]; Chang et al. [7]; Li et al. [8]; Gao et al. [18]; Gao et al. [19]). When a weak layer exists (the strength of a layer is relatively weak) in a slope, the slip surface of the soil slides along the weak layer (Griffiths and Marquez [14]; Huang et al. [16]). Therefore, the weak layer governs the failure mechanism and the conventional rotational log-spiral failure mechanism is not suitable. Farzaneh et al. [20] developed a rotational-translational mechanism to solve the bearing capacity problems. Recently, Huang et al. [16] proposed a rotational-translational mechanism, which contains three rigid blocks, for a slope with a weak layer, as shown in Figure 1. The velocity of block $b$ is $v_{b}$, while the angular velocities of block $a$ and block $c$ are $\omega_{a}$ and $\omega_{c}$, respectively. Every point on discontinuous surfaces FN and O'M should satisfy the kinematically admissible velocity. Thus, computational effort is needed to determine those discontinuous surfaces, which might become an issue when deriving upper-bound limit solution in seismic analysis.

In this study, a translational failure mechanism was developed. As shown in Figure 2, the rigid rotational blocks $a$ and $c$ (see Figure 1) were replaced by continuous deformation regions $a$ and $c$ (log-spiral shear zones), consisting of a sequence of $n$ rigid triangles. These triangles satisfy the

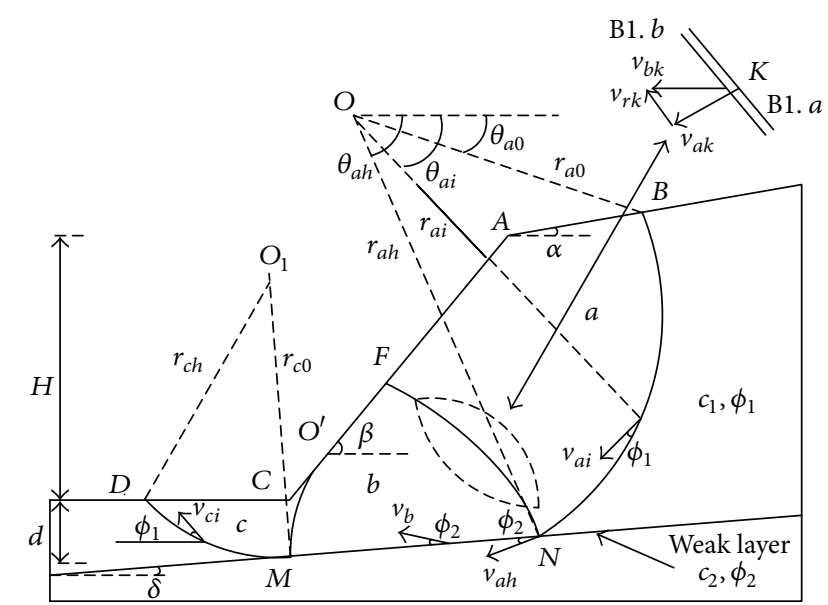

FIGURE 1: Rotational-translational mechanism (after Huang et al. 2013 [16]).

admissible velocity field of every discontinuous surface. The number of triangles in those regions can be increased without extra analytical effort. Those continuous deformation regions $a$ and $c$ are separated by the translational rigid block $b$. Due to velocity discontinuities between adjacent blocks, energy is dissipated at their interfaces. The velocity hodographs are shown in Figure 3, in which $v_{b}$ is the velocity of rigid block $b$; $v_{a h}$ is the velocity at the end point $N$ of the log-spiral shear zone $a ; v_{c 0}$ is the velocity at the start point $M$ of the log-spiral shear zone $c$. The directions of the velocities incline at an angle $\psi=\varphi$ with the corresponding discontinuous surfaces. These velocities constitute a kinematically admissible velocity field.

According to the theory of limit analysis, the slopes will collapse if the rate of work performed by external forces and soil weight exceeds the energy dissipation rate for any assumed kinematically admissible failure mechanism. The rate of external work due to the soil weight in this translational failure mechanism is the sum of different elements:

$$
W^{\gamma}=W_{a}+W_{b}+W_{c}
$$

The external work due to the self-weight of the log-spiral shear zones $a$ and $c$ can be expressed as

$$
\begin{aligned}
W_{a} & =\int_{\theta_{a 0}}^{\theta_{a h}} m_{a} \cdot g \cdot v_{a} \cdot \cos \theta d \theta \\
& =\int_{\theta_{a 0}}^{\theta_{a h}} \frac{1}{2} \gamma\left(r_{a}^{2}-l_{a}^{2}\right) \cos \theta \\
\cdot & v_{a 0} \exp \left(\left(\theta-\theta_{a 0}\right) \cdot \tan \varphi_{1}\right) d \theta, \\
W_{c} & =\int_{\theta_{c 0}}^{\theta_{c h}} m_{c} \cdot g \cdot v_{c} \cdot \cos \theta d \theta \\
& =\int_{\theta_{c 0}}^{\theta_{c h}} \frac{1}{2} \gamma\left(r_{c}^{2}-l_{c}^{2}\right) \cos \theta \\
& \cdot v_{c 0} \exp \left(\left(\theta-\theta_{c 0}\right) \cdot \tan \varphi_{1}\right) d \theta .
\end{aligned}
$$




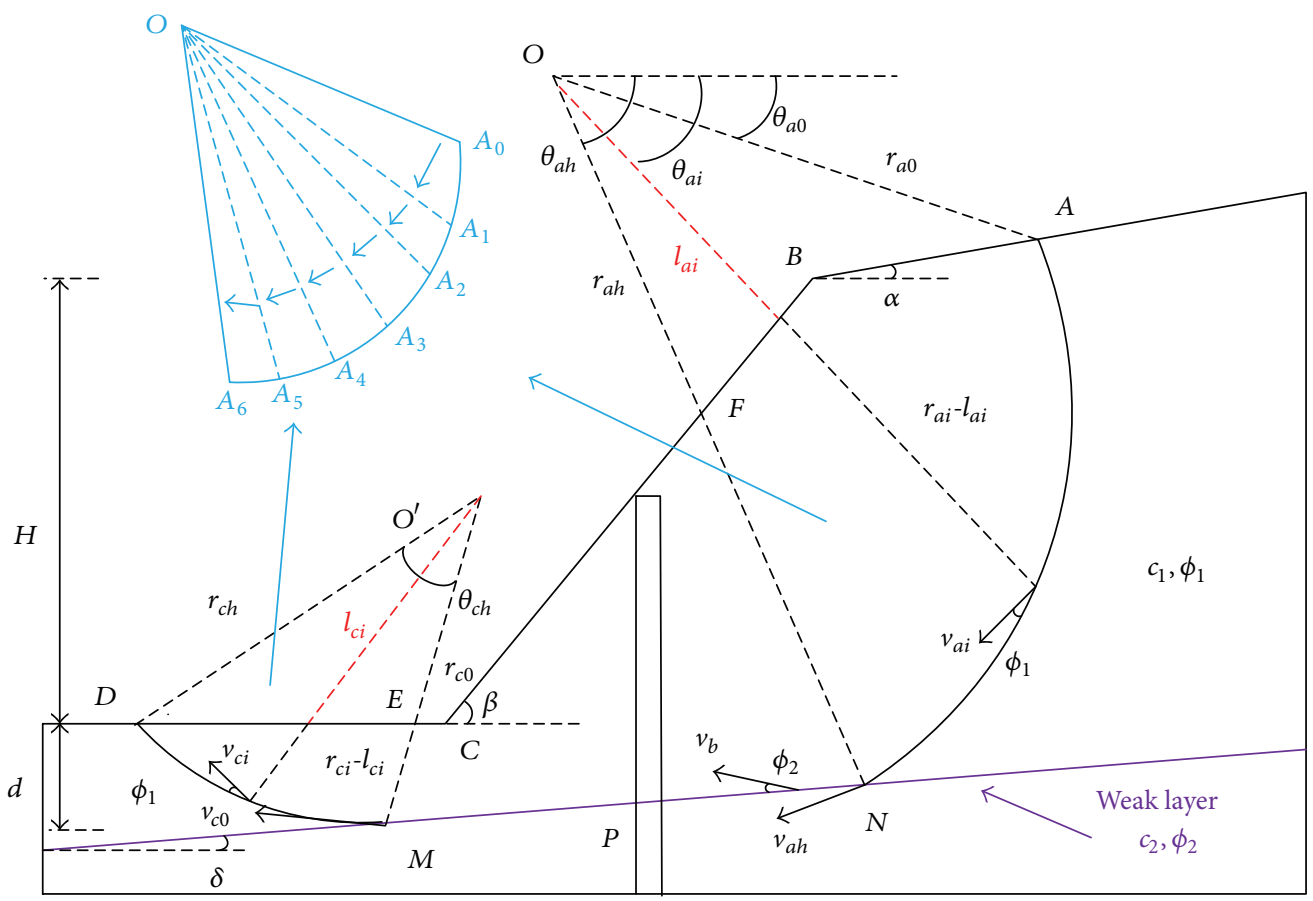

FIgURE 2: Translational collapse mechanism for pile-reinforced slopes with a weak layer.

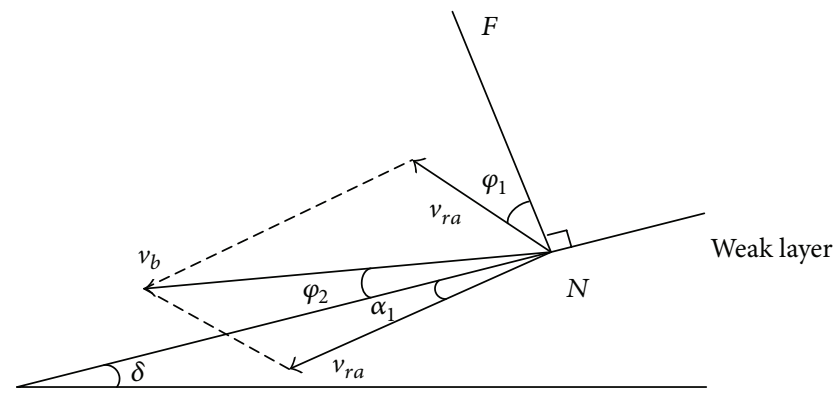

(a)

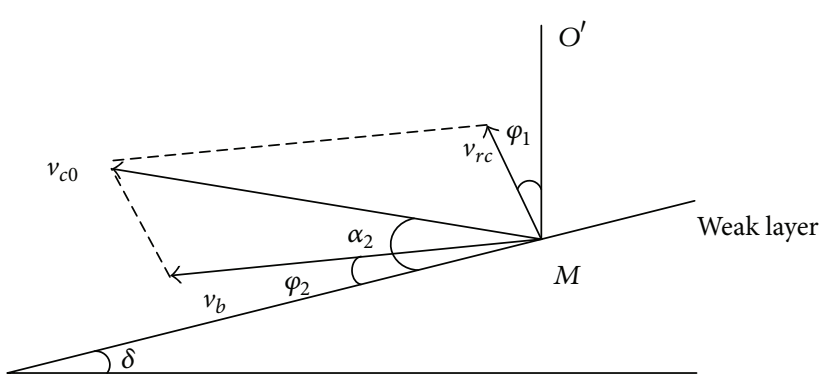

(b)

FIGURE 3: Velocity hodographs at points (a) $M$ and (b) $N$.

The external work due to the self-weight of block $b$ can be written as

$$
W_{b}=m_{b} g \sin \left(\delta-\varphi_{2}\right) v_{b}=\gamma S_{b} \sin \left(\delta-\varphi_{2}\right) v_{b}
$$

where $r_{a}$ and $r_{c}$ are the radius of the log-spiral with respect to angles $\theta_{a 0}$ and $\theta_{c 0}$, respectively; $\gamma$ is the unit weight of the soils; $\varphi_{1}$ and $\varphi_{2}$ are the internal friction angles of the soils, respectively. $l_{a}$ and $l_{c}$ are shown in Figure $2 ; S_{b}$ is the area of block $b$.

Once the slope with a weak layer is subjected to seismic loads, the rate of the inertial force should be considered in the energy balance equation. The collapse mechanism is considered as an incipient mechanism and does not influence the shaking mode (Li et al. [8]; He et al. [12]). The effects of the seismic loads acting on the potential sliding mass are represented by horizontal forces applied to the center of soil mass. The rate of external work due to the inertial force is the sum of different elements

$$
W^{s}=W_{a}^{s}+W_{b}^{s}+W_{c}^{s}
$$

The external work due to the inertial force of log-spiral shear zones $a$ and $c$ can be expressed as

$$
\begin{aligned}
W_{a}^{s} & =k \int_{\theta_{a 0}}^{\theta_{a h}} m_{a} \cdot g \cdot v_{a} \cdot \sin \theta d \theta \\
& =k \int_{\theta_{a 0}}^{\theta_{a h}} \frac{1}{2} \gamma\left(r_{a}^{2}-l_{a}^{2}\right) \sin \theta \\
& \cdot v_{a 0} \exp \left(\left(\theta-\theta_{a 0}\right) \cdot \tan \varphi_{1}\right) d \theta,
\end{aligned}
$$




$$
\begin{aligned}
W_{c}^{s} & =k \int_{\theta_{c 0}}^{\theta_{c h}} m_{c} \cdot g \cdot v_{c} \cdot \sin \theta d \theta \\
& =k \int_{\theta_{c 0}}^{\theta_{c h}} \frac{1}{2} \gamma\left(r_{c}^{2}-l_{c}^{2}\right) \sin \theta \\
& \cdot v_{c 0} \exp \left(\left(\theta-\theta_{c 0}\right) \cdot \tan \varphi_{1}\right) d \theta .
\end{aligned}
$$

The external work due to the inertial force of block $b$ can be written as

$$
W_{b}^{s}=k m_{b} g \cos \left(\delta-\varphi_{2}\right) v_{b}=k \gamma S_{b} \cos \left(\delta-\varphi_{2}\right) v_{b} .
$$

The technique of stabilizing piles, which has been proven as an efficient approach to enhancing the stability of a slope, is widely adopted in geotechnical engineering practice. It provides lateral force for resistance to the sliding of a slope (He et al. [21]). The total energy dissipation of pile-reinforced slopes is the sum of $D^{c}$ and $D^{p}$, which can be expressed as

$$
D=D^{p}+D^{c}
$$

where $D^{p}$ is the rate of work dissipation caused by the piles; $D^{c}$ is the rate of energy dissipation of soil cohesion. To calculate the effect of the piles, a lateral force is assumed to act at the potential failing surface. The energy dissipation rate can be written as

$$
D^{p}=F_{p} v_{i} \cos \left(\delta-\varphi_{2}\right),
$$

where $F_{p}$ is the lateral force exerted by the piles per unit thickness of the sliding mass; $v_{i}$ is the velocity of the sliding soil.

The theoretical method proposed by Ito and Matsui [22] was adopted in this analysis to calculate lateral force $F_{p}$ acting on the piles. The lateral force $F_{p}$ can be expressed as follows:

$$
\begin{aligned}
F_{p} & =A c H\left(\frac { 1 } { N _ { \varphi } \operatorname { t a n } \varphi } \left\{\operatorname { e x p } \left[\frac{D_{1}-D_{2}}{D_{2}} N_{\varphi} \tan \varphi\right.\right.\right. \\
& \left.\left.\cdot \tan \left(\frac{\pi}{8}+\frac{\varphi}{4}\right)\right]-2 N_{\varphi}^{1 / 2} \tan \varphi-1\right\} \\
& \left.+\frac{2 \tan \varphi+2 N_{\varphi}^{1 / 2}+N_{\varphi}^{-1 / 2}}{N_{\varphi}^{1 / 2} \tan \varphi+N_{\varphi}-1}\right)-c H\left(D_{1}\right. \\
& \left.\cdot \frac{2 \tan \varphi+2 N_{\varphi}^{1 / 2}+N_{\varphi}^{-1 / 2}}{N_{\varphi}^{1 / 2} \tan \varphi+N_{\varphi}-1}-2 D_{2} N_{\varphi}^{-1 / 2}\right) \\
& +\frac{\gamma H^{2}}{2 N_{\varphi}}\left\{A \exp \left[\frac{D_{1}-D_{2}}{D_{2}} N_{\varphi} \tan \varphi \tan \left(\frac{\pi}{8}+\frac{\varphi}{4}\right)\right]\right. \\
& \left.-D_{2}\right\}
\end{aligned}
$$

where $D_{1}$ is the center-to-center spacing between neighboring piles; $D_{2}$ is the clear spacing between neighboring piles; $H$ is the height of the unstable soil layer from the ground surface to the slip surface; $N_{\varphi}=\tan ^{2}(\pi / 4+\varphi / 2)$; $A=D_{1}\left(D_{1} / D_{2}\right)\left(N_{\varphi}^{1 / 2} \tan \varphi+N_{\varphi}-1\right)$. It can be observed that the lateral force increases as the ratio $D_{2} / D_{1}$ decreases. The method assumes that the piles are rigid and the soil surrounding the piles deforms plastically. Li et al. [8] reported that when the spacing $D_{2} / D_{1}$ is below approximately $0.5-$ 0.6 , piles act like a continuous wall and soil around the piles cannot reach the plastic state. Thus, values of $D_{2} / D_{1}$ larger than 0.5 were used in this study.

The rate of internal energy dissipation $D^{c}$ induced by soil cohesion in the collapse mechanism can be calculated as follows:

$$
D^{c}=D_{a}+D_{\mathrm{FN}}+D_{b}+D_{\mathrm{EM}}+D_{c}
$$

in which

$$
\begin{aligned}
D_{b} & =l_{\mathrm{MN}} c_{2} v_{b} \cos \varphi_{2}, \\
D_{\mathrm{FN}} & =c_{1} l_{\mathrm{FN}} \sin 2 \varphi_{2} v_{b}, \\
D_{\mathrm{EM}} & =c_{1} l_{\mathrm{EM}} \sin \left(\varphi_{1}-\varphi_{2}\right) v_{b} .
\end{aligned}
$$

Energy dissipation in the radial shear regions $a$ and $c$ is the sum of the rate of dissipation along the discontinuity surfaces and the rate of dissipation within the continually deforming log spiral shear regions. As shown in Figure 2, energy dissipation takes place along the radial lines $O A_{0}$, $O A_{1}, O A_{2}$, and so forth because all the triangles move as rigid blocks in directions that make an angle $\varphi_{1}$ with the discontinuity surfaces. Energy is also dissipated on the discontinuity surfaces $A_{0}, A_{1}, A_{2}, A_{3}, A_{4}, A_{5}$, and $A_{6}$ between the material at rest and the material in motion. The energy dissipation along the discontinuity surfaces is the cohesion multiplied by the relative velocities and the length of discontinuity lines in the soil mass

$$
\begin{aligned}
& D_{a} \\
& =c_{1} \int_{\theta_{a 0}}^{\theta_{a h}}\left(2 r_{a 0}-l_{a}\right) \cdot v_{a 0} \exp \left(2\left(\theta-\theta_{a 0}\right) \cdot \tan \varphi_{1}\right) d \theta, \\
& D_{c} \\
& =c_{1} \int_{\theta_{c 0}}^{\theta_{c h}}\left(2 r_{c 0}-l_{c}\right) \cdot v_{c 0} \exp \left(2\left(\theta-\theta_{c 0}\right) \cdot \tan \varphi_{1}\right) d \theta .
\end{aligned}
$$

To determine the critical yield acceleration coefficient $k_{c}$, at which the slope will be in the limit state, $k_{c}$ can be calculated by equating the rate of internal energy dissipation to the external rate of work; that is,

$$
W^{\gamma}+W^{s}=D^{p}+D^{c}
$$
lated as
The critical yield acceleration coefficient $k_{c}$ can be calcu- 


$$
k_{c}=\frac{D^{p}+D^{c}-W^{\gamma}}{\int_{\theta_{a 0}}^{\theta_{a h}} m_{a} \cdot g \cdot v_{a} \cdot \sin \theta d \theta+m_{b} g \cos \left(\delta-\varphi_{2}\right) v_{b}+\int_{\theta_{c 0}}^{\theta_{c h}} m_{c} \cdot g \cdot v_{c} \cdot \sin \theta d \theta},
$$

where $m_{a}, m_{b}$, and $m_{c}$ are the masses of different elements; $g$ is the gravitational acceleration. The minimum critical yield acceleration coefficient was obtained with respect to the parameters of $\theta_{a 0}, \theta_{a h}, \theta_{c 0}, \theta_{c h}, v_{a h} / v_{b}$, and $v_{c 0} / v_{b}$.

\section{Assessment of Cumulative Displacement of Pile-Reinforced Slopes with a Weak Layer Subjected to Seismic Loads}

Once the horizontal earthquake acceleration exceeds the critical value, the displacement of the soil mass begins to accumulate. Newmark's sliding block technique was adopted to evaluate the cumulative displacement of the slope subjected to seismic loads. The classical sliding block technique involves only one block; this method was employed by other researchers to evaluate the stability of a slope under the rotational failure mechanism in both $2 \mathrm{D}$ and $3 \mathrm{D}$ problems (e.g., Chang et al. [7]; Li et al. [8]; He et al. [12]). This technique was adopted in this study for the translational mechanism of a pile-reinforced slope with a weak layer. When the earthquake acceleration is exceeded, the sliding block starts to accelerate with acceleration items $\ddot{u}_{a}, \ddot{u}_{b}$, and $\ddot{u}_{c}$; thus, balancing the work rate equation yields

$$
\begin{aligned}
W_{\gamma}+W_{s}= & D_{c}+D_{p}+\int_{\theta_{a 0}}^{\theta_{a h}} m_{a} v_{a} \ddot{u}_{a} d \theta \\
& +\int_{\theta_{c 0}}^{\theta_{c h}} m_{c} v_{c} \ddot{u}_{c} d \theta+m_{b} v_{b} \ddot{u}_{b} .
\end{aligned}
$$

According to Newmark's analytical approach [6], the sliding mass moves only downhill, irrespective of the direction of the earthquake acceleration. Earthquake acceleration varies during the process of shaking. The slope movement will occur when the seismic coefficient $k$ exceeds its critical yield acceleration coefficient $k_{c}$. The velocity of the failing mass increases from zero and reaches its peak value when the earthquake acceleration decreases to a certain critical value. After that, the failing mass will still move until the acceleration drops back down to some level below the critical yield acceleration coefficient $k_{c}$ (He et al. [12]). The acceleration can be integrated to calculate the velocity of the failing soil mass, while cumulative displacement can be estimated by integrating velocity (Newmark [6]). Subtracting (14) from (15), the acceleration can be expressed as

$$
\begin{aligned}
& g\left(k-k_{c}\right)\left(\int_{\theta_{a 0}}^{\theta_{a h}} m_{a} \cdot \frac{v_{a}}{v_{b}} \cdot \sin \theta d \theta+m_{b} \cos \left(\delta-\varphi_{2}\right)\right. \\
& \left.\quad+\int_{\theta_{c 0}}^{\theta_{c h}} m_{c} \cdot \frac{v_{c}}{v_{b}} \cdot \sin \theta d \theta\right) \\
& =\ddot{u}_{b}\left(\int_{\theta_{a 0}}^{\theta_{a h}} m_{a} \cdot \frac{v_{a}}{v_{b}} \cdot \frac{\ddot{u}_{a}}{\ddot{u}_{b}} d \theta+\int_{\theta_{c 0}}^{\theta_{c h}} m_{c} \cdot \frac{v_{c}}{v_{b}} \cdot \frac{\ddot{u}_{c}}{\ddot{u}_{b}} d \theta\right. \\
& \left.\quad+m_{b}\right) .
\end{aligned}
$$

To satisfy the kinematical admissibility, the ratios $\ddot{u}_{a h} / \ddot{u}_{b}$ and $\ddot{u}_{b} / \ddot{u}_{c 0}$ are the same as $v_{a h} / v_{b}$ and $v_{b} / v_{c 0}$ (Michalowski and You [11]), which can be expressed as

$$
\begin{aligned}
& \frac{v_{a h}}{v_{b}}=\frac{\ddot{u}_{a h}}{\ddot{u}_{b}}=A_{a}, \\
& \frac{v_{c 0}}{v_{b}}=\frac{\ddot{u}_{c 0}}{\ddot{u}_{b}}=A_{c} .
\end{aligned}
$$

Thus, the acceleration $\ddot{u}_{b}$ becomes

$$
\ddot{u}_{b}=g\left(k-k_{c}\right) \frac{\int_{\theta_{a 0}}^{\theta_{a h}} m_{a} \cdot A_{a} \cdot \sin \theta d \theta+m_{b} \cos \left(\delta-\varphi_{2}\right)+\int_{\theta_{c 0}}^{\theta_{c h}} m_{c} \cdot A_{c} \cdot \sin \theta d \theta}{\int_{\theta_{a 0}}^{\theta_{a h}} m_{a} A_{a}^{2} d \theta+m_{b}+\int_{\theta_{c 0}}^{\theta_{c h}} m_{c} A_{c}^{2} d \theta}=g\left(k-k_{c}\right) C .
$$

This expression indicates that soil acceleration is not related to the piles directly but the piles affect the failure mechanism and the critical yield acceleration coefficient $k_{c}$. The failure mechanism tends to reach below the toe of slopes due to the location of the weak layer. The horizontal displacement in the toe of slopes must be determined, though the analysis allows for calculation of the displacement of any point in the failing mass; that is,

$$
\begin{aligned}
u_{b(x)} & =\cos \left(\delta-\varphi_{2}\right) \iint_{t} \ddot{u}_{b} d t d t \\
& =C \cos \left(\delta-\varphi_{2}\right) \iint_{t} g\left(k-k_{c}\right) d t d t .
\end{aligned}
$$




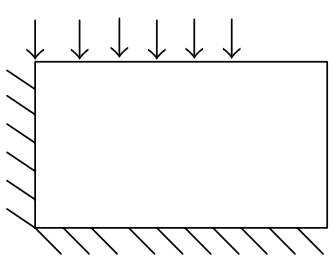

(a)

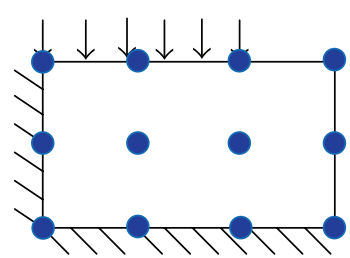

(b)

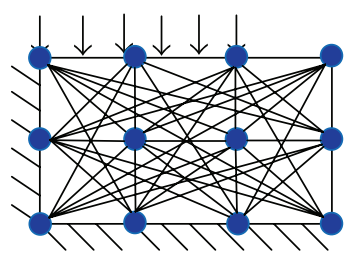

(c)

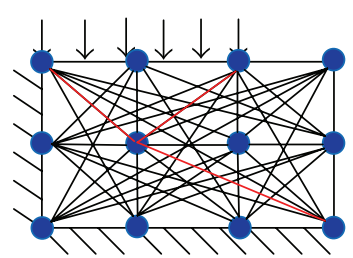

(d)

FIGURE 4: Procedure of DLO to determine critical layout of slip-lines: (a) starting problem; (b) discretization of soil by nodes; (c) interconnection of nodes with potential discontinuities; (d) identification of critical subset of potential discontinuities by optimization (adapted from Smith and Gilbert [25]).

Coefficient $C$ was calculated based on the proposed translational mechanism. Additionally, the geometry of the slope and soil properties are contained in coefficient $C$, which is involved in the optimization of the collapse mechanism. The double integral in (19) is dependent on the earthquake acceleration record and the critical yield acceleration coefficient of the pile-reinforced slope.

\section{Comparison with Other Results}

Before the proposed translational mechanism was used for the analysis of cumulative displacement, it was validated using other solutions with respect to the static factor of safety. The Monte Carlo technique (Hammersley and Handscomb [23]) was employed for determining the critical collapse mechanism and the critical yield acceleration coefficient $k_{c}$. This technique is efficient in the search for the critical slip surface of slope stability analysis (e.g., He et al. [12]). To obtain a more reliable solution, efficient searches are needed (500,000 trials).

For verification of the proposed solution, the factor of safety predicted by the proposed limit analysis was compared with the results obtained by using different computational approaches. The solutions from the available literature and the numerical program LimitState: GEO (LimitState [24]) were selected for comparison. LimitState: GEO, which has been successfully adopted in evaluating slope stability and other geotechnical problems (e.g., Smith and Gilbert [25, 26]; Leshchinsky and Ambauen [27]), employs the Discontinuity Layout Optimization (DLO) technique to evaluate the critical failure mechanisms and associated upper-bound solution. As shown in Figure 4, it automatically searches for the critical failure surface in a soil mass that is at a limit state and has no need to preassume a critical collapse mechanism. Thus, it can be considered as a useful tool for ensuring the accuracy of the failure mechanism assumed in this study. Two typical cases of slopes with a weak thin layer were selected. The comparison is based on natural slopes without reinforcement.

Slope $A$, shown in Figure 5, is taken from Huang et al. [16]. A nonhomogeneous slope for $\beta=45^{\circ}$ has a height of $6 \mathrm{~m}$ and is built from a soil characterized by $\varphi_{1}=10^{\circ}$, $c_{1}=20 \mathrm{kPa}$, and $\gamma_{1}=20 \mathrm{kN} / \mathrm{m}^{3}$, with a $0.5 \mathrm{~m}$ thick weak layer characterized by $\varphi_{2}=5^{\circ}, c_{2}=12 \mathrm{kPa}$, and $\gamma_{2}=20 \mathrm{kN} / \mathrm{m}^{3}$. To validate the derived equation and the random search approach, the factor of safety computed by
TABLE 1: Comparison of the results of factor of safety (slope $A$ ).

Factor of safety

\begin{tabular}{lccc}
$\begin{array}{l}\text { Present } \\
\text { study }\end{array}$ & LA (Huang et al. [16]) & FEM (Huang et al. [16]) & DLO \\
\hline 1.31 & 1.27 & 1.28 & 1.25 \\
\hline
\end{tabular}

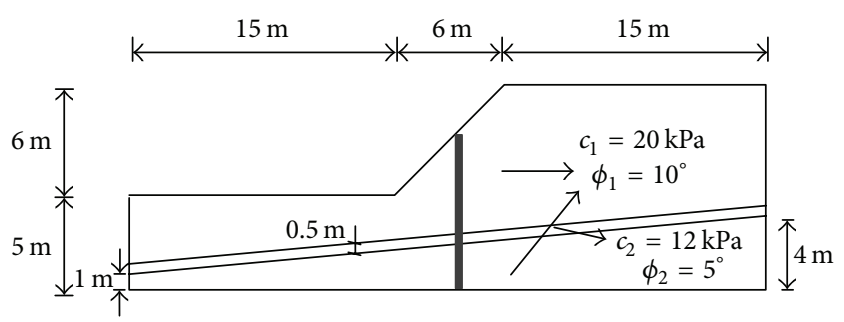

FIGURE 5: Schematic diagram of slope $A$.

the translational mechanism was compared with the results obtained by Huang et al. [16] and the DLO. The comparison is shown in Table 1. It can be observed that the calculated values from the proposed translational mechanism and the existing rotational-translational mechanism (Huang et al. $[16])$ were slightly higher than the result from DLO $(\approx 4 \%$ and $1 \%$, resp.). The computed critical failure mechanism, that is, panhandling failure mechanism that partially penetrates the weak layer, can be observed in Figure 7. While the values of the factor of safety are quite close, the slip surface from Huang et al. [16] is not similar to that of DLO. In their study, the velocities of point $N$ in block $a$ and point $M$ in the block $c$ were fixed. It means that the ratios $v_{a h} / v_{b}$ and $v_{c 0} / v_{b}$ were not considered in the optimization, which contributes to the deviation of the slip surface. On the other hand, the predicted critical failure surface from the translational mechanism was similar to that of DLO, which proves the reliability of the random search approach.

Slope $B$ is a nonhomogeneous slope underlain by a thin weak layer, as shown in Figure 6 (Fredlund and Krahn [13]). A $1: 2$ slope has a height of $10 \mathrm{~m}$, and the soil properties are $\varphi_{1}=$ $20^{\circ}, c_{1}=29 \mathrm{kPa}$, and $\gamma_{1}=18.8 \mathrm{kN} / \mathrm{m}^{3}$, with a weak layer $\left(\varphi_{2}=\right.$ $10^{\circ}, c_{2}=0 \mathrm{kPa}$, and $\left.\gamma_{2}=18.8 \mathrm{kN} / \mathrm{m}^{3}\right)$ in the foundation of the slope. Fredlund and Krahn [13] compared several methods for calculating this slope. The limit equilibrium solutions (Spencer's method and Janbu's rigorous method) published 
TABLE 2: Comparison of the results of factor of safety (slope $B$ ).

\begin{tabular}{lccc}
\hline \multicolumn{4}{c}{ Factor of safety } \\
Present study & Spencer's method (Fredlund and Krahn [13]) & Janbu's rigorous method (Fredlund and Krahn [13]) & DLO \\
\hline 1.36 & 1.377 & 1.432 & 1.32 \\
\hline
\end{tabular}

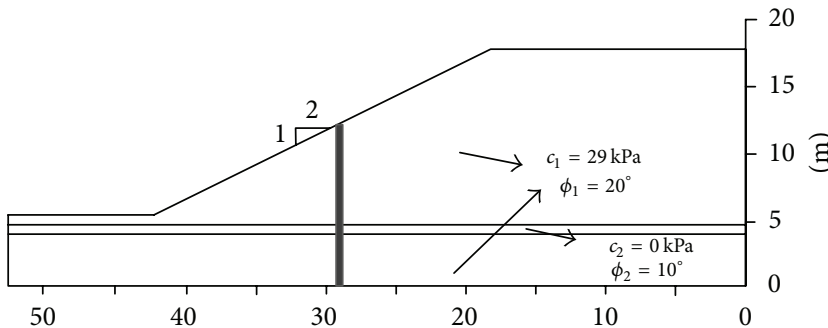

Figure 6: Schematic diagram of slope $B$.

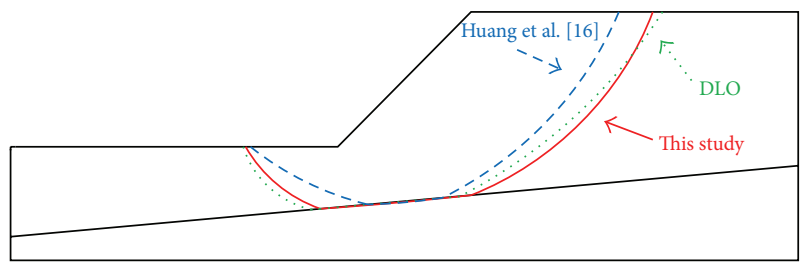

FIGURE 7: Comparison of failure mechanisms of slope $A$.

by Fredlund and Krahn [13] were selected in this section, whereas the results of DLO were also compared. As shown in Table 2, the present upper-bound solution yields a factor of safety of 1.36, which is in the vicinity of that of other methods. Figure 8 shows the comparison of the predicted failure mechanism between the upper-bound solution and limit equilibrium method for slope $B$. Furthermore, the critical slip surface from the limit analysis is reasonably consistent with that of DLO but quite different from the one of Fredlund and Krahn [13]. In the limit equilibrium method (Fredlund and Krahn [13]), the analytical parameters (e.g., failure radii, number of slices, and interslice forces) require high computational effort. The discrepancy of the slip surfaces may be attributed to the limitation of computational constraints in the limit equilibrium. Thus, the factor of safety is sensitive to the slip surface.

The agreement is satisfactory, in terms of both factor of safety and the location of its associated critical failure surface. The computed results show good agreement with the values obtained by other approaches. With the confidence gained through the validation, the next section examines the cumulative displacement of pile-reinforced slopes with a weak thin layer.

\section{Example}

The cumulative displacements of slopes with a weak layer subjected to seismic loads were evaluated by using Newmark's analytical approach. Moreover, the effects of the stabilizing piles on the cumulative displacement were also compared.

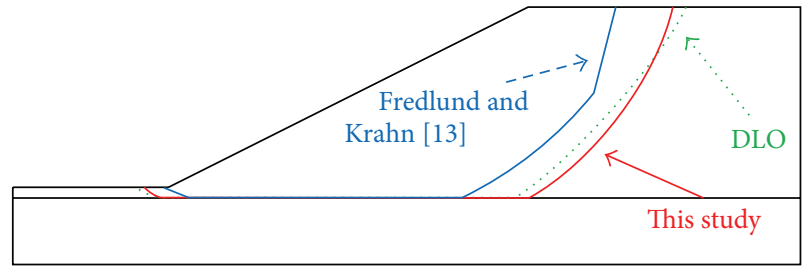

FIGURE 8: Comparison of failure mechanisms of slope $B$.

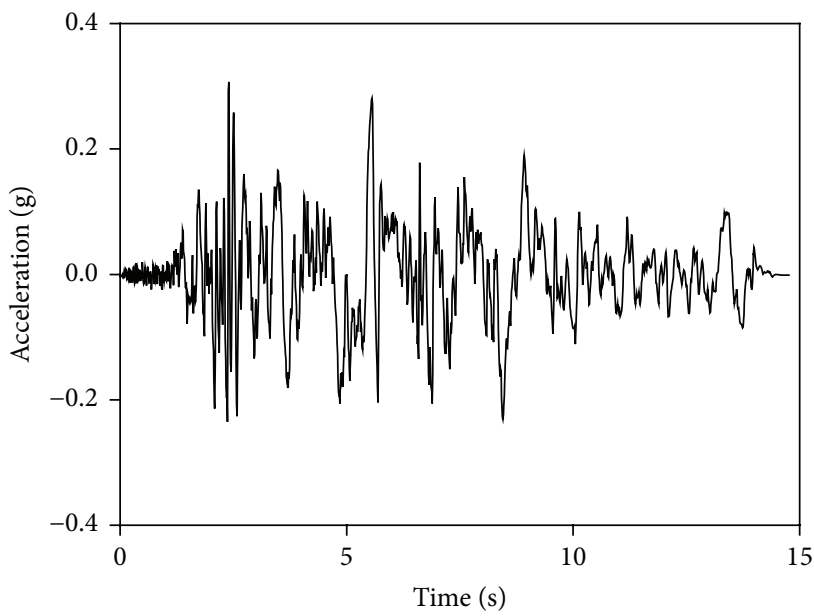

FIGURE 9: Acceleration records of Imperial Valley-06 Earthquake $(\mathrm{PGA}=0.307 \mathrm{~g}$, duration $=14.76 \mathrm{~s})$.

The properties and geometries of the slope are shown in Figures 5 and 6. Ho [15] employed the finite element method (FEM) to draw the conclusion that the middle portion of the slope is the optimal pile position for slopes with a weak layer. Thus, the pile was assumed to be in the middle of the slope to assess the seismic displacement in this study.

The displacement of a structure due to seismic loads depends on the different real earthquake acceleration time histories. To make the results useful, the cases selected in this study were evaluated for different earthquake acceleration records. As shown in Figures 9-11, three typical earthquake records (He et al. [12]) were used to estimate the seismic displacements. Figure 9 is the Imperial Valley-06 Earthquake records, with peak earthquake acceleration (PGA) of $0.307 \mathrm{~g}$ and a record duration of $14.76 \mathrm{~s}$. Figure 10 is the Kobe Earthquake record, with PGA $=0.345 \mathrm{~g}$ and a record duration of 20 s. Figure 11 is the Parkfield-02 Earthquake record, with PGA $=0.373 \mathrm{~g}$ and a duration of $21.07 \mathrm{~s}$. The constant time interval of the Imperial Valley-06 and Kobe Earthquake records is $0.01 \mathrm{~s}$, while the constant time interval of the Parkfield02 Earthquake record is $0.005 \mathrm{~s}$. These earthquake records were used one after another to assess the performance of 
TABLE 3: Results of cumulative displacement for slopes with a weak layer with and without piles.

\begin{tabular}{|c|c|c|c|c|c|c|c|c|c|}
\hline & & \multicolumn{2}{|c|}{ Without piles } & \multicolumn{6}{|c|}{ With piles } \\
\hline & & Slope $A$ & Slope $B$ & Slope $A$ & Slope $B$ & Slope $A$ & Slope $B$ & Slope $A$ & Slope $B$ \\
\hline$D_{2} / D_{1}$ & & - & - & 0.9 & 0.9 & 0.85 & 0.85 & 0.8 & 0.8 \\
\hline$k_{c}$ & & 0.145 & 0.122 & 0.257 & 0.172 & 0.273 & 0.190 & 0.294 & 0.211 \\
\hline \multirow{3}{*}{$u_{x}(\mathrm{~cm})$} & Imperial Valley-06 & 16.89 & 29.49 & 0.324 & 8.54 & 0.068 & 5.422 & 0.006 & 2.907 \\
\hline & Kobe & 42.43 & 67.59 & 1.673 & 23.20 & 1.002 & 15.17 & 0.415 & 8.428 \\
\hline & Parkfield-02 & 49.13 & 68.45 & 8.845 & 30.99 & 6.490 & 23.06 & 3.955 & 16.75 \\
\hline
\end{tabular}

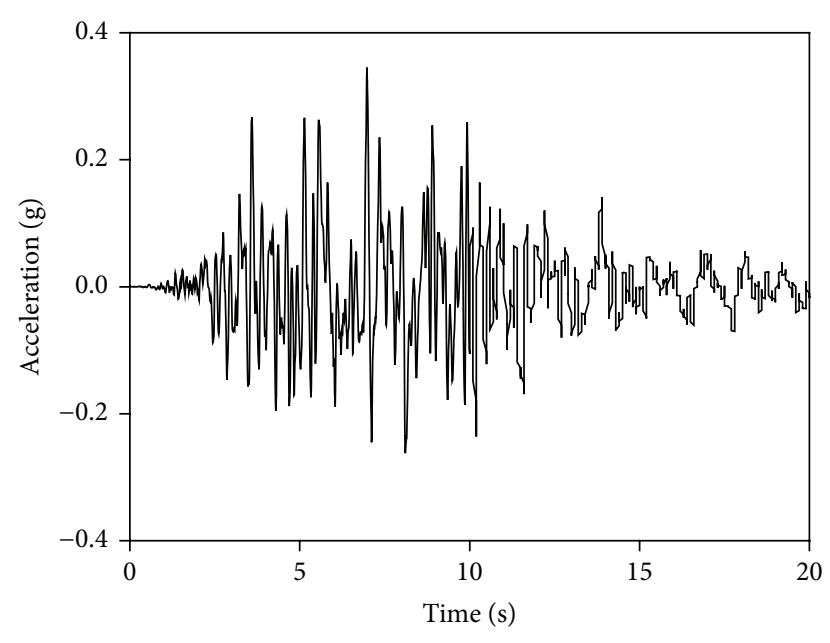

FIGURE 10: Acceleration records of Kobe Earthquake (PGA = $0.345 \mathrm{~g}$, duration $=20 \mathrm{~s}$ ).

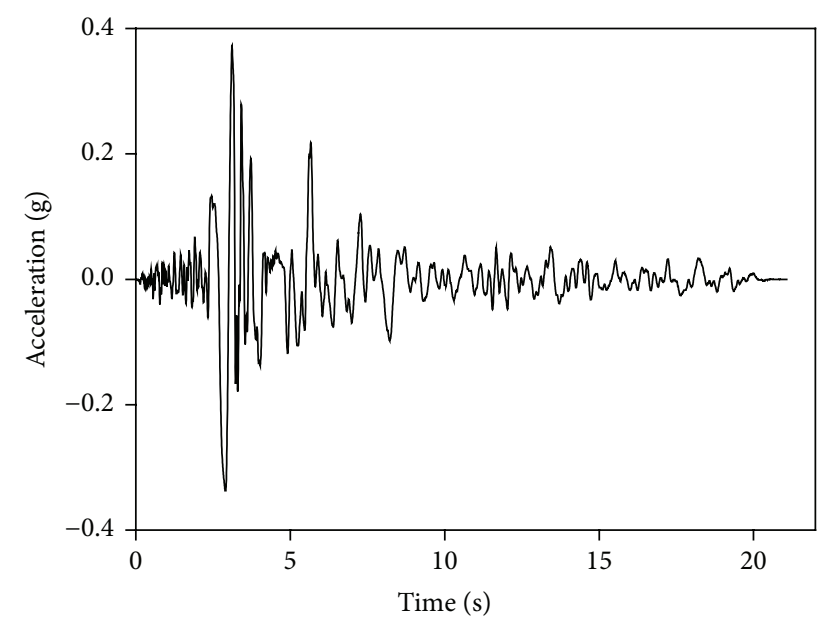

Figure 11: Acceleration records of Parkfield-02 (CA) Earthquake $(\mathrm{PGA}=0.373 \mathrm{~g}$, duration $=21.07 \mathrm{~s})$.

the slopes (with/without reinforcement) subjected to seismic loads. The results are listed in Table 3. Calculations of the slope displacements are illustrated for the above two example slopes.

The seismic loads of the Imperial Valley-06 Earthquake, Kobe Earthquake, and Parkfield-02 Earthquake were exerted on the slopes, resulting in displacements of $16.89 \mathrm{~cm}$, $42.43 \mathrm{~cm}$, and $49.13 \mathrm{~cm}$ for slope $A$ and $29.49 \mathrm{~cm}, 67.59 \mathrm{~cm}$, and $68.45 \mathrm{~cm}$ for slope $B$, respectively. The maximum horizontal displacements of the slopes increase with the peak earthquake acceleration of the earthquake experiences. Michalowski and You [11] introduced a displacement criterion for a slope subjected to seismic loads. In the criterion, a slope is allowed to move with a small displacement. The maximum horizontal displacement at the toe $u_{x}$ should be smaller than $0.005 \mathrm{H}$. According to the results, the displacements of those two cases exceed this criterion. The piles must be embedded to improve the performance of the slope, and the different ratios of spacing between neighboring piles $D_{2} / D_{1}$ of $0.80,0.85$, and 0.90 were compared in this study. The ratio $D_{2} / D_{1}$ of 0.80 should be used for the Parkfield02 Earthquake with $\mathrm{PGA}=0.373 \mathrm{~g}$, while the ratio $D_{2} / D_{1}$ of 0.90 is sufficient for slope $A$ under the Imperial Valley-06 Earthquake. Generally, the results show the efficiency of piles in limiting the deformation of the slope under earthquake loading, especially for slope $A$. This may be because the location of the weak layer in slope $A$ is deeper. Thus, the height of the portion of piles above the sliding surface was relatively higher, resulting in larger lateral force $F_{p}$ of the piles. For Parkfield-02 Earthquake record $(\mathrm{PGA}=0.373 \mathrm{~g})$, the reduced displacement ratios for slope $B(H=10 \mathrm{~m})$ are $45 \%$, $66 \%$, and $75.5 \%$ with different $D_{2} / D_{1}$ ratios, respectively. $\mathrm{Li}$ et al. [8] evaluated the effect of piles $\left(D_{2} / D_{1}=0.6\right)$ on the displacement of homogeneous slopes with a height of $13.7 \mathrm{~m}$. Under a seismic load with PGA $=0.4 \mathrm{~g}$, the reduced ratios of displacement ranging from $68 \%$ to $86 \%$ were reported in their study. For Parkfield-02 Earthquake record, He et al. [12] reported that the piles $\left(D_{2} / D_{1}=0.85\right)$ reduced the cumulative displacement ratio of a homogeneous slope $(H=$ $12 \mathrm{~m}$ ) by about $50 \%$. This comparison further proves that the $D_{2} / D_{1}$ ratio significantly influences the deformation of a slope. Comparing with the homogeneous slope, the piles contribute to the larger benefit in slopes with a weak layer. This is because the pile reduces the depth of slip surface in homogeneous slopes (Ausilio et al. [28]), leading to the limiting effect of pile lateral force.

\section{Conclusion}

Previous studies of slopes with a weak layer have focused on the static factor of safety. The seismic performance of pile-reinforced slopes with a weak layer is evaluated in this study. Based on the upper-bound theorem of limit analysis, a translational mechanism is developed to determine the critical yield acceleration coefficient and the accumulative 
displacement. The rigid rotational blocks in the rotationaltranslational failure mechanism proposed by Huang et al. [16] were replaced by continuous deformation regions $a$ and $c$, with a sequence of $n$ rigid triangles. The factor of safety and failure mechanism, evaluated by using the proposed translational failure mechanism, exhibit excellent agreement with other solutions of the available literature and the DLO technique, which proves the accuracy of the proposed failure mechanism.

To account for the presence of piles, the plastic theory is employed to calculate the lateral force and energy dissipation of the piles. Newmark's analytical procedure is adopted to assess the cumulative displacement of pile-reinforced slopes with a weak layer subjected to seismic loads. Two typical cases existing in the available literature are shown to illustrate the influence of an earthquake on the displacements of slopes. The earthquake experiences and the peak earthquake acceleration have a significant influence on the displacement of the slope. Stabilizing piles can be regarded as an efficient approach in reducing the cumulative displacement of slopes with a weak thin layer, and reducing the spacing of piles is needed for large earthquake acceleration.

\section{Competing Interests}

The authors declare that they have no competing interests.

\section{Acknowledgments}

This research was funded by the National Natural Science Foundation of China (Grant no. 51378345). The authors would also like to thank the China Scholarship Council for their support.

\section{References}

[1] H. B. Seed, K. L. Lee, and I. M. Idriss, "Analysis of the sheffield dam failure," Journal of the Soil Mechanics and Foundations Division, ASCE, vol. 95, no. 6, pp. 1453-1490, 1969.

[2] H. B. Seed, "Considerations in the earthquake-resistant design of earth and rockfill dams," Geotechnique, vol. 29, no. 3, pp. 215263,1979 .

[3] W. F. Chen, "Plasticity in soil mechanics and landslides," Journal of the Engineering Mechanics Division, vol. 106, no. 3, pp. 443464,1980 .

[4] H. I. Ling, D. Leshchinsky, and E. B. Perry, "Seismic design and performance of geosynthetic-reinforced soil structures," Geotechnique, vol. 47, no. 5, pp. 933-952, 1997.

[5] R. L. Michalowski, "Soil reinforcement for seismic design of geotechnical structures," Computers and Geotechnics, vol. 23, no. 1-2, pp. 1-17, 1998.

[6] N. M. Newmark, "Effects of earthquakes on dams and embankments," Géotechnique, vol. 15, no. 2, pp. 139-160, 1965.

[7] C.-J. Chang, W. F. Chen, and J. T. P. Yao, "Seismic displacements in slopes by limit analysis," Journal of Geotechnical Engineering, vol. 110, no. 7, pp. 860-874, 1984.

[8] X. Li, S. He, and Y. Wu, "Seismic displacement of slopes reinforced with piles," Journal of Geotechnical and Geoenvironmental Engineering, vol. 136, no. 6, pp. 880-884, 2010.
[9] R. L. Michalowski, "Displacements of multiblock geotechnical structures subjected to seismic excitation," Journal of Geotechnical and Geoenvironmental Engineering, vol. 133, no. 11, pp. 14321439, 2007.

[10] H. I. Ling and D. Leshchinsky, "Effects of vertical acceleration on seismic design of geosynthetic-reinforced soil structures," Géotechnique, vol. 48, no. 3, pp. 347-373, 1998.

[11] R. L. Michalowski and L. You, "Displacements of reinforced slopes subjected to seismic loads," Journal of Geotechnical and Geoenvironmental Engineering, vol. 126, no. 8, pp. 685-694, 2000.

[12] Y. He, H. Hazarika, N. Yasufuku, Z. Han, and Y. Li, “Threedimensional limit analysis of seismic displacement of slope reinforced with piles," Soil Dynamics and Earthquake Engineering, vol. 77, pp. 446-452, 2015.

[13] D. G. Fredlund and J. Krahn, "Comparison of slope stability methods of analysis," Canadian Geotechnical Journal, vol. 14, no. 3, pp. 429-439, 1977.

[14] D. V. Griffiths and R. M. Marquez, "Three-dimensional slope stability analysis by elasto-plastic finite elements," Géotechnique, vol. 57, no. 6, pp. 537-546, 2007.

[15] I.-H. Ho, "Numerical study of slope-stabilizing piles in undrained clayey slopes with a weak thin layer," International Journal of Geomechanics, vol. 15, no. 5, 2014.

[16] M. Huang, H. Wang, D. Sheng, and Y. Liu, "Rotationaltranslational mechanism for the upper bound stability analysis of slopes with weak interlayer," Computers and Geotechnics, vol. 53, pp. 133-141, 2013.

[17] X. Lu, H. Wang, and M. Huang, "Upper bound solution for the face stability of shield tunnel below the water table," Mathematical Problems in Engineering, vol. 2014, Article ID 727964, 11 pages, 2014.

[18] Y. Gao, F. Zhang, G. H. Lei, D. Li, Y. Wu, and N. Zhang, "Stability charts for 3D failures of homogeneous slopes," Journal of Geotechnical and Geoenvironmental Engineering, vol. 139, no. 9, pp. 1528-1538, 2013.

[19] Y. Gao, S. Yang, F. Zhang, and B. Leshchinsky, "Threedimensional reinforced slopes: evaluation of required reinforcement strength and embedment length using limit analysis," Geotextiles and Geomembranes, vol. 44, no. 2, pp. 133-142, 2016.

[20] O. Farzaneh, N. Ganjian, and F. Askari, "Rotation-translation mechanisms for upper-bound solution of bearing capacity problems," Computers and Geotechnics, vol. 37, no. 4, pp. 449455, 2010.

[21] Y. He, H. Hazarika, N. Yasufuku, J. Teng, Z. Jiang, and Z. Han, "Estimation of lateral force acting on piles to stabilize landslides," Natural Hazards, vol. 79, no. 3, pp. 1981-2003, 2015.

[22] T. Ito and T. Matsui, "Methods to estimate lateral force acting on stabilizing piles," Soils and Foundations, vol. 15, no. 4, pp. 43-59, 1975.

[23] J. M. Hammersley and D. C. Handscomb, Monte Carlo Method, Methuen, London, UK, 1964.

[24] LimitState, LimitState: Geo Manual v 3.0, LimitState, Sheffield, UK, 2013

[25] C. C. Smith and M. Gilbert, "Identification of rotational failure mechanisms in cohesive media using discontinuity layout optimisation," Geotechnique, vol. 63, no. 14, pp. 1194-1208, 2013.

[26] C. C. Smith and M. Gilbert, "Application of discontinuity layout optimization to plane plasticity problems," Proceedings of The Royal Society of London A, vol. 463, no. 2086, pp. 2461-2484, 2007. 
[27] B. Leshchinsky and S. Ambauen, "Limit equilibrium and limit analysis: comparison of benchmark slope stability problems," Journal of Geotechnical and Geoenvironmental Engineering, vol. 141, no. 10, Article ID 04015043, 2015.

[28] E. Ausilio, E. Conte, and G. Dente, "Stability analysis of slopes reinforced with piles," Computers and Geotechnics, vol. 28, no. 8, pp. 591-611, 2001. 


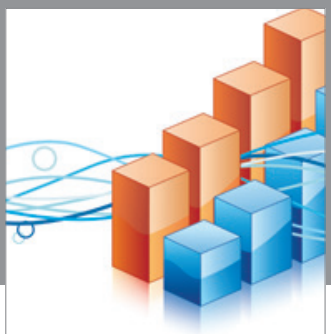

Advances in

Operations Research

vatem alat4

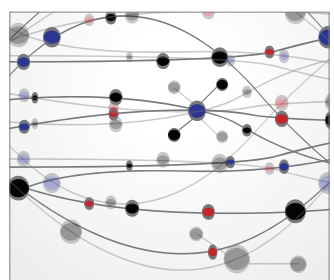

\section{The Scientific} World Journal
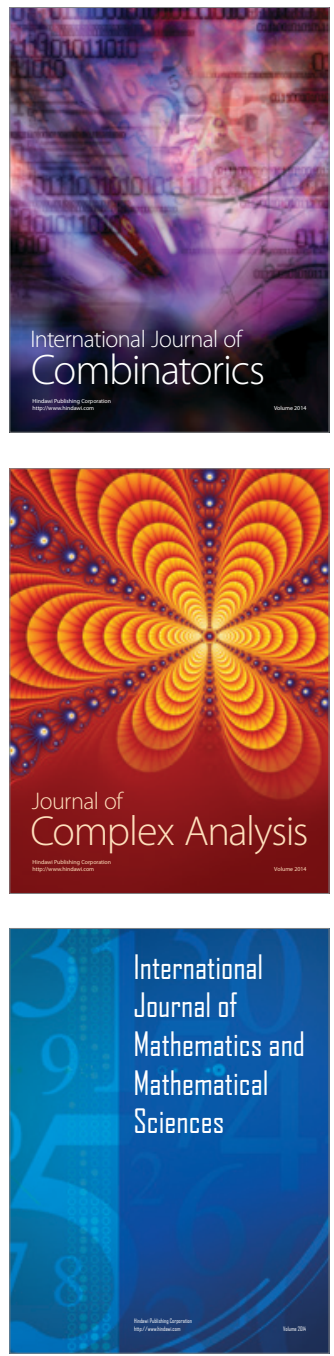
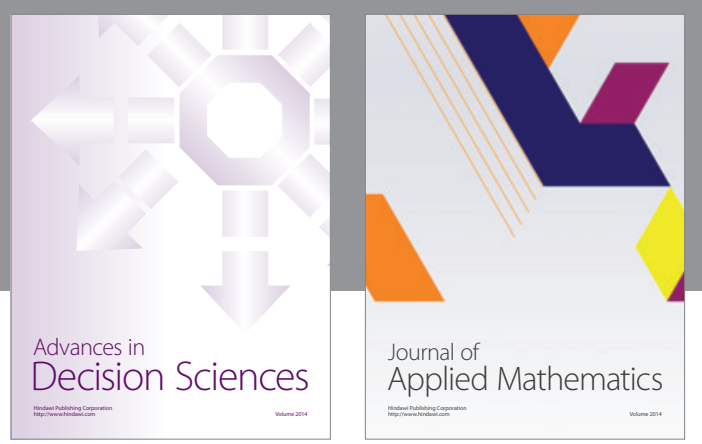

Algebra

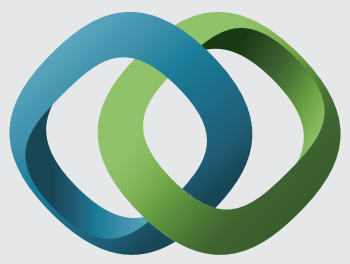

\section{Hindawi}

Submit your manuscripts at

http://www.hindawi.com
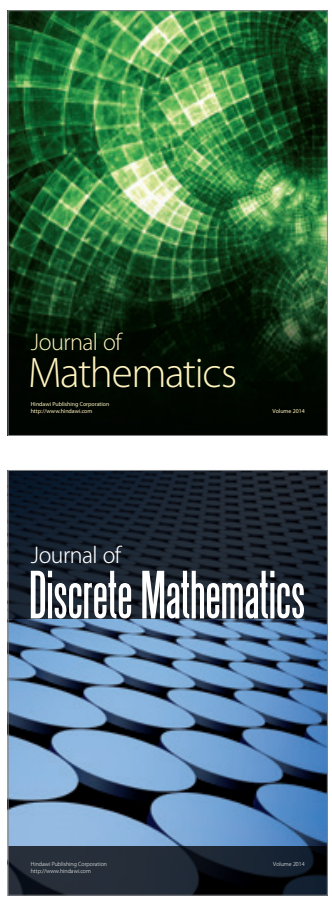

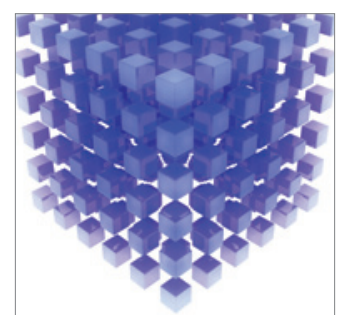

Mathematical Problems in Engineering
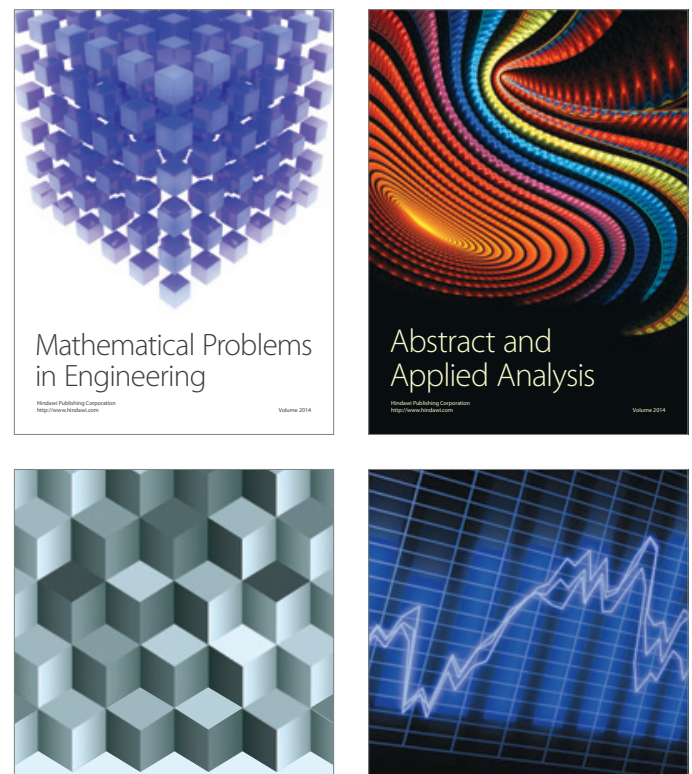

Journal of

Function Spaces

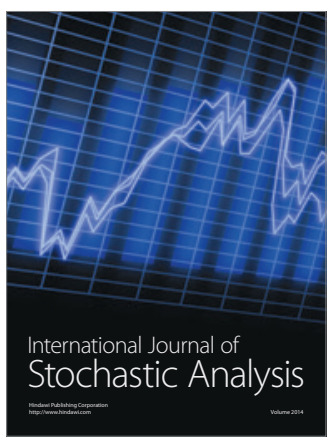

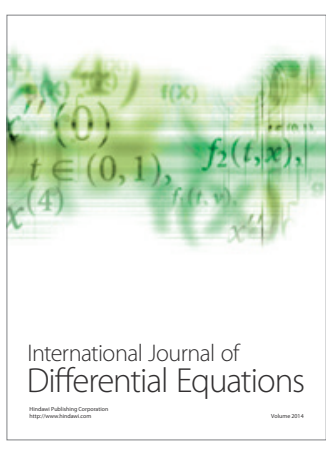
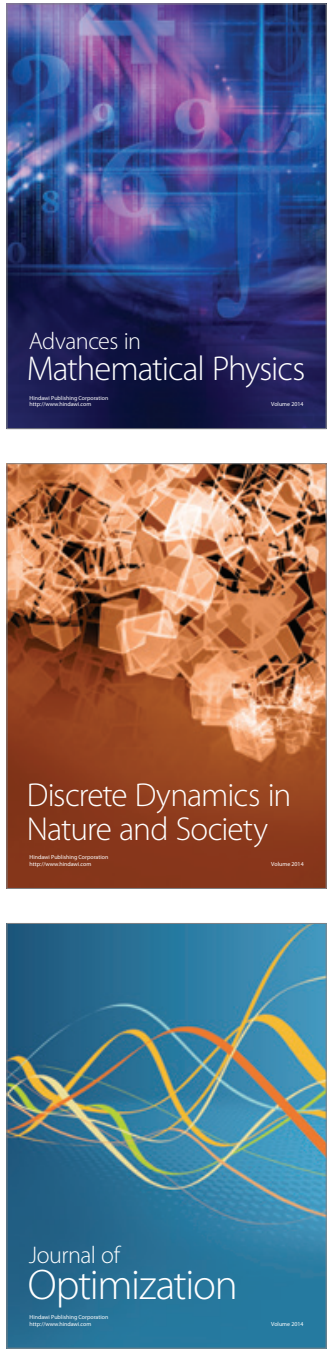